Pacific

Journal of

Mathematics

\title{
RULINGS OF LEGENDRIAN KNOTS AS SPANNING SURFACES
}

TAMÁS KÁLMÁN 


\title{
RULINGS OF LEGENDRIAN KNOTS AS SPANNING SURFACES
}

\author{
TAMÁs KÁLMÁN
}

\begin{abstract}
Each ruling of a Legendrian link can be naturally treated as a surface. For knots, the ruling is 2 -graded if and only if the surface is orientable. For 2-graded rulings of homogeneous (in particular, alternating and positive) knots, we show that the genus of this surface is at most the genus of the knot. While this is not true in general, we do prove that the canonical genus of any knot is an upper bound for the genera of its 2-graded rulings.
\end{abstract}

\section{Introduction}

A Legendrian knot in $\mathbb{R}_{x y z}^{3}$ is a smooth embedding of $S^{1}$ that is always tangent to the 2-planes of the standard contact structure $\xi=\operatorname{ker}(\mathrm{d} z-y \mathrm{~d} x)$, and a link is a finite disjoint union of knots. We consider Legendrian knots up to Legendrian isotopy, that is homotopy through Legendrian knots. The most basic, "classical" invariants of Legendrian isotopy are the Thurston-Bennequin number $t b$, rotation number $r$, and smooth type [Etnyre 2005]. (Of course, any invariant of smooth isotopy is also an invariant of Legendrian isotopy.) In this paper, we work with the so-called ruling invariants [Pushkar' and Chekanov 2005; Fuchs 2003]. In the spirit of "maximum Thurston-Bennequin number", we introduce a new smooth knot invariant $\rho$ called ruling genus (Definition 3.4) and study its properties.

The beginning of Legendrian knot theory dates back to Bennequin's proof [1983] of the famous inequality

$$
t b(L)+|r(L)| \leq 2 g(L)-1,
$$

where $L$ is an arbitrary Legendrian knot and $g(L)$ is its Seifert genus. (For elements of knot theory, we refer the reader to [Cromwell 2004].) Our main goal is to strengthen the relationship between the genus and Legendrian invariants by proving the following:

Theorem 1.1. The genus of any 2-graded ruling of the Legendrian knot L is less than or equal to the canonical genus of L. In short, $\rho \leq \tilde{g}$ for any smooth knot type.

MSC2000: 53D12, 57M25.

Keywords: knot, Legendrian knot, genus, canonical genus, ruling, spanning surface. 
Motivated by the theory of generating functions, rulings (also know as decompositions) were introduced by Pushkar' and Chekanov [2005] and independently by Fuchs [2003]. Rulings and the idea that a genus may be associated to them are central to this paper. However the discussion of these notions is deferred to Section 2. Recall that the canonical genus (also known as diagram genus) $\tilde{g}$ is the minimum of the genera of spanning surfaces obtained using Seifert's algorithm on diagrams of the given knot. The proof of Theorem 1.1, at the end of Section 3, is an easy application of a result of Rutherford [2006] and Morton's inequality [1986] that the $z$-degree of the Homfly polynomial bounds the canonical genus from below.

Because for homogeneous knots (a class of knots that includes all alternating and positive knots) the canonical genus agrees with the genus [Cromwell 2004, section 7.6], the following is immediate.

Corollary 1.2. The genus of any 2-graded ruling of the homogeneous Legendrian $k n o t L$ is less than or equal to the genus of $L$. In other words, $\rho \leq g$ for homogeneous knots.

In addition to homogeneous knots, the statement of the corollary holds for all prime knots up to 13 crossings; see Section 4. It also holds for connected sums of the aforementioned knots by the additivity of genus and Proposition 4.1. It does not, however, hold in general.

Example 1.3. There exist (prime) knots whose genus is less than the maximum of the genera of their 2-graded rulings. Two examples are shown in Figure 1. One of these $\left(14 n_{22180}\right.$ in the Hoste-Thistlethwaite table) is a Whitehead double of the trefoil, but the other $\left(14 n_{19265}^{*}\right)$ is not a satellite knot (thus it is hyperbolic). Both are of genus 1 and both possess 2-graded rulings with genera up to 2. The genus 2 ruling is unique and $\mathbb{Z}$-graded (see Definition 2.1) for both. This shows that Corollary 1.2 fails for nonhomogeneous knots even if we restrict the statement to $\mathbb{Z}$-graded rulings.

The paper is organized as follows. In Section 2 we review rulings, their gradedness and genera, and establish some relations of these to the topology of the ruling. In Section 3 we review Rutherford's work and prove Theorem 1.1. In Section 4 we claim further properties of the ruling genus and explain how the two knots of Example 1.3 were found. Section 5 contains some further ideas and open questions.

\section{Rulings and their genera}

The front diagram of a Legendrian link $L \subset \mathbb{R}_{x y z}^{3}$ is its projection onto the $x z$ plane. For generic $L$, it has an equal number of left and right cusps (with respect to the $x$-direction), and the rest of the diagram consists of immersed strands with 

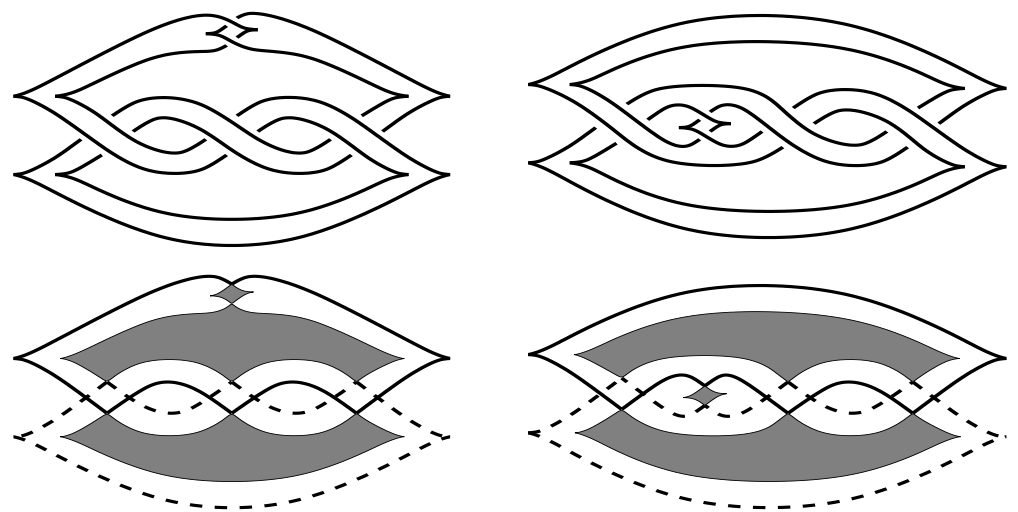

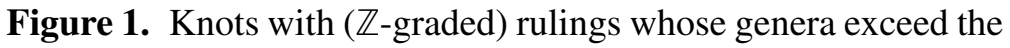
knot's genus. (These front diagrams are nongeneric but no switch is vertically aligned with a nonswitching crossing.)

no vertical tangents that connect a left cusp to a right cusp. These may intersect but may not be tangent to each other. A Maslov potential [Pushkar' and Chekanov 2005; Fuchs 2003] is a locally constant $\mathbb{Z}_{2 r}$-valued ${ }^{1}$ function defined along these strands with the following property: at each cusp, of the two strands that join there, the (locally) upper one has potential that is 1 higher than that of the other strand.

The index of a crossing in a front diagram is the difference (in $\mathbb{Z}_{2 r}$ ) of the Maslov potentials of the two intersecting strands. (The potential of the lower strand is to be subtracted from that of the upper one.) We adopt the convention that when a front diagram is oriented, strands traveling to the right (increasing $x$ ) have even Maslov potential. This implies, even for multicomponent links, that a crossing has even index if and only if it is positive. For the following definition [Pushkar' and Chekanov 2005; Fuchs 2003], we assume that the front diagram of the Legendrian is generic in that no pair of crossings share the same $x$-coordinate.

Definition 2.1. A ruling is a partial smoothing of a front diagram $f$ where certain crossings, called switches, are replaced by a pair of arcs as in Figure 2. The resulting diagram is a union of eyes, that is pairs of arcs connecting the same left and right cusps. The interiors of the two arcs forming an eye are disjoint, contain no cusps, and do not meet at switches. We assume that in the vertical ( $x=$ const.) slice of the diagram through each switch, the two eyes that meet at the switch fit one of the three configurations in the middle of Figure 2.

\footnotetext{
${ }^{1}$ The rotation $r$, up to a sign, of a Legendrian link is defined as the greatest common divisor of the rotations of its constituent components.
} 

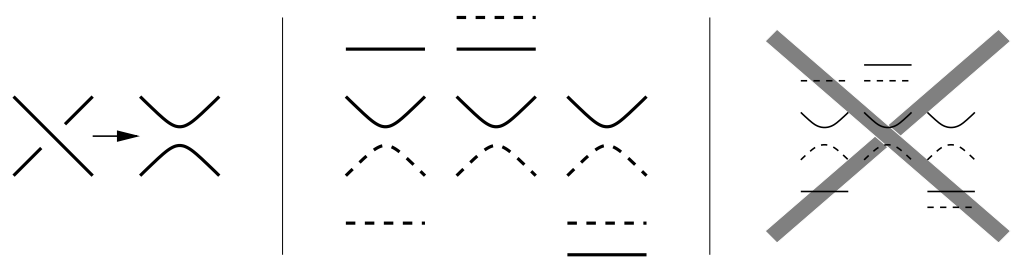

Figure 2. Allowed and disallowed configurations for switches of rulings.

The notion above is also known as an ungraded ruling. If we assert that all switches be of even index, we get 2-graded rulings. ${ }^{2}$ The set of these objects will be denoted by $\Gamma_{2}(f)$. Finally, $\mathbb{Z}$-graded rulings are defined only when $r=0$; they are those in which each switch has index 0 .

Figure 1 shows two examples. Note that in any ruling, the number of eyes equals the number of left cusps.

Definition 2.2. The eyes bound disks and with the addition of twisted bands at the switches, we may consider the ruling as a surface. ${ }^{3}$ This may be referred to as the surface associated to the ruling, but sometimes we will simply identify the ruling and the surface. The genus of a ruling is that of its associated surface.

This surface does not come with an embedding into $\mathbb{R}^{3}$ though. (In [Ekholm et al. $\geq 2008$ ], we will associate to it an immersed exact Lagrangian in $B^{4}$.) In fact it follows from the results of this paper that such an embedding often does not exist. But first, let us make several simple observations.

Proposition 2.3. The surface associated to a 2-graded ruling is orientable. If the surface associated to an ungraded ruling of a Legendrian link is orientable, then the link has an orientation so that the ruling is 2-graded.

Proof. Consider the Maslov potential on the front diagram. The entire top arc of each eye in a 2-graded ruling is either on odd or on even potential and the entire bottom arc has the opposite parity. Switches connect odd arcs to odd arcs and even arcs to even arcs. Hence if we give "odd top" eyes one orientation of the projection plane and to "even top" eyes the opposite orientation, then (by an examination of Figure 2) this will extend continuously over the bands.

For the other claim, simply induce an orientation of the Legendrian from that of its ruling. By doing so, all switches will be positive crossings.

\footnotetext{
${ }^{2}$ In the multicomponent case, orientation of the diagram and our "even-right" convention for the Maslov potential are important.

${ }^{3}$ Compare with Seifert's construction and with the graph $K_{D}$ of [Pushkar' and Chekanov 2005, $\S 6]$.
} 
Now the following is obvious. We remark that in [Rutherford 2006], 2-graded rulings are referred to as oriented rulings.

Corollary 2.4. For front diagrams of (single-component) knots, a ruling is 2graded if and only if its associated surface is orientable.

Remark 2.5. Ungraded, that is not-necessarily-orientable versions of Theorem 1.1 and Corollary 1.2 fail even for alternating knots. The knot $5_{1}$ (the positive $(5,2)$ torus knot) bounds an embedded Möbius band, thus having unorientable genus 1, and it also has a ruling of oriented genus 2 (that is, unoriented genus 4).

\section{Proof of Theorem 1.1}

We may classify rulings according to their genera. In fact the quantity

$$
\theta(\eta)=\text { number of eyes }- \text { number of switches, }
$$

introduced in [Pushkar' and Chekanov 2005], is just the Euler characteristic of the surface associated to the ruling $\eta$.

The counts of ungraded, 2-graded, and $\mathbb{Z}$-graded rulings with a given genus (or Euler characteristic) are Legendrian isotopy invariants [Pushkar' and Chekanov 2005]. The sequence of these counts for all genera is called the complete ruling invariant. In the $\mathbb{Z}$-graded case, the complete ruling invariant is very effective in distinguishing Legendrian knots with the same classical invariants. By contrast, the ungraded and 2-graded complete ruling invariants are determined by the smooth type and the Thurston-Bennequin number. This is true by the two main theorems in [Rutherford 2006]. In particular, in the 2-graded case Rutherford proves the following.

Theorem 3.1 [Rutherford 2006]. Let $P_{L}(v, z)$ denote the Homfly polynomial of the Legendrian knot L, and let $Q_{L}(z)$ be the coefficient of $v^{t b(L)+1}$ in $P_{L}(v, z)$. Then

$$
Q_{L}(z)=\sum_{\eta \in \Gamma_{2}(L)} z^{2 g(\eta)} .
$$

Except for replacing the indeterminate $a$ with $v^{-1}$, we formulate the Homfly polynomial as in [Rutherford 2006]. In particular, it is normalized so that $P=1$ is associated to the unknot. Our changing (in the exponent of $z$ ) of Rutherford's

$$
j(\eta)=\text { number of switches }- \text { number of left cusps }+1=-\theta(\eta)+1
$$

to $2 g(\eta)$ is valid by the first claim in Proposition 2.3.

There is an important context to Theorem 3.1. For a smooth knot type $K$, let $T B(K)$ denote the maximum Thurston-Bennequin number of $K$, that is the maximum of $t b(L)$ over all Legendrian representatives $L$ of $K$. Let $e$ be the lowest $v$-degree in the Homfly polynomial of $K$. Then, 
Theorem 3.2 [Franks and Williams 1987; Morton 1986]. $T B(K)+1 \leq e$.

Comparing the last two theorems, we obtain:

Corollary 3.3 [Rutherford 2006]. Only Legendrians realizing TB of their smooth type may have 2-graded rulings.

Much of the rest of the paper is devoted to the study of the following concept.

Definition 3.4. For a knot $K$, we define the ruling genus $\rho(K)$ as the supremum of the genera of all 2-graded rulings of all Legendrian representatives of $K$.

This is either a finite value (and in that case a maximum) or $-\infty$ (when no Legendrian representative has 2-graded rulings).

Proposition 3.5. For any knot type $K$, we have

$$
\rho(K)>-\infty \text { if and only if } T B(K)=e-1 .
$$

Any Legendrian representative $L$ of $K$ with $t b(L)=T B(K)$ is such that the highest genus attained by its 2-graded rulings is $\rho(K)$.

Proof. If $\rho(K)>-\infty$ then $K$ has a Legendrian representative $L$ which has 2graded rulings. Then by Theorem 3.1 we have

$$
t b(L)+1 \geq e,
$$

hence $T B(K)+1 \geq e$, and by Theorem 3.2 ,

$$
T B(K)+1=e .
$$

Conversely, if $K$ has a Legendrian representative $L$ so that $t b(L)+1=e$, then $L$ has 2 -graded rulings by Theorem 3.1 and so $\rho(K)>-\infty$.

The other claim is also easy if we put together Corollary 3.3 and the fact that by Theorem 3.1, the genus distribution of 2-graded rulings depends only on $t b$ and the Homfly polynomial.

Corollary 3.6. The ruling genus $\rho$ is a function of TB and the Homfly polynomial.

In particular, if we know that $T B=e-1$, namely that $\rho \neq-\infty$, then we may directly read $\rho$ off of the Homfly polynomial as one half of the highest $z$-exponent among terms of $P$ that have the lowest $v$-exponent. On the other hand, deciding whether $\rho(K)=-\infty$ is essentially equivalent to knowing $T B(K)$. At the time of preparing this paper, that is still a hard problem in general.

With the notation of Definition 3.4, Corollary 1.2 simply asserts that

$$
\rho(K) \leq g(K)
$$

for all homogeneous $K$, and Theorem 1.1 asserts that

$$
\rho(K) \leq \tilde{g}(K) \text { for all } K .
$$




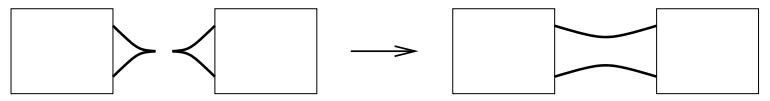

Figure 3. Connected sum operation.

Proof of Theorem 1.1. From Theorem 3.1, it is obvious that the ruling genus $\rho$ is at most half of the $z$-degree of the Homfly polynomial, which in turn is a well-known lower bound [Morton 1986, Theorem 2] for the canonical genus of the knot.

\section{Other properties of the ruling genus}

The next statement essentially follows from [Pushkar' and Chekanov 2005, Proposition 11.2]. It could also be derived from known properties of $T B$ and $P$ via Corollary 3.6.

Proposition 4.1. The ruling genus is additive for connected sums:

$$
\rho\left(K_{1} \# K_{2}\right)=\rho\left(K_{1}\right)+\rho\left(K_{2}\right) .
$$

Proof. Choose front diagrams for $K_{1}$ and $K_{2}$ with maximal Thurston-Bennequin number. One may form their connected sum as in Figure 3. Assume without loss of generality that the Maslov potentials near the two merging cusps match. Then it is plain to see that with any of the three notions of Definition 2.1, the set of rulings for the new diagram is the Cartesian product of the two old sets. The claim follows at once from Proposition 3.5.

One finds several sufficient conditions that imply $\rho \leq g$ in terms of polynomial knot invariants. ${ }^{4}$ These are listed in the two propositions below. As before, let $e$ denote the minimum $v$-degree of the Homfly polynomial $P_{K}(v, z)$ of $K$. Versions of (iii) and (iv) in the following list were also published in [Ng 2006].

Proposition 4.2. For a smooth knot type $K$, any of the following conditions implies that $K$ does not have Legendrian representatives with 2-graded rulings, and hence that $\rho(K)=-\infty$.

(i) "Khovanov beats Homfly":

$$
e \geq 2+\min \left\{k \mid \bigoplus_{i-j=k} H K h^{i, j}(K) \neq 0\right\},
$$

where $H K h^{i, j}(K)$ is the Khovanov homology group of $K$ in bigrading $(i, j)$.

\footnotetext{
${ }^{4}$ For a long time, I tried to prove that $\rho \leq g$ for all knots. Whitehead doubles, having genus 1 , are natural candidates for a counterexample; see Example 1.3. However I initially dismissed them, having misunderstood a claim in [Fuchs 2003].
} 
(ii) "Kauffman beats Homfly": the minimum v-degree of the Kauffman polynomial of $K$ is less than $e$.

(iii) "Negative counts": there is an integer $i$ so that the coefficient $p_{e, i}$ of $v^{e} z^{i}$ in the Homfly polynomial is negative.

(iv) "Subset failure": the minimum v-degree in the Kauffman polynomial is e or more, but there is an integer $i$ so that if $f_{e, i}$ is the coefficient of $v^{e} z^{i}$ in the Dubrovnik version of the Kauffman polynomial, then $0 \leq p_{e, i} \leq f_{e, i}$ fails to hold.

Proof. Recall from Proposition 3.5 that 2-graded rulings exist if and only if $t b+1=$ $e$ for some Legendrian representing $K$. This is what we have to prevent.

In the first two cases, we separate $t b+1$ and $e$ by inequalities. Claim (i) is a direct consequence of the so-called Khovanov bound of the Thurston-Bennequin number [Ng 2005]. Claim (ii) follows in the same straightforward way from the Kauffman bound [Rudolph 1990].

In the other two cases, we assume $t b+1=e$ and find a contradiction with Rutherford's results. Claim (iii) is obvious from Theorem 3.1. To prove the last statement, one needs to compare Theorem 3.1 with Rutherford's other main theorem [2006], which says that the coefficients $f_{t b+1, i}$ count ungraded rulings of some given $(\theta=1-i$, to be exact) Euler characteristic. Now the contradiction is obvious from the fact that 2 -graded rulings are also ungraded.

Let $M$ denote the maximum $z$-degree that appears in the Homfly polynomial of $K$ in a monomial that also contains $v^{e}$. With this, either $\rho=M / 2$ or $\rho=-\infty$. (We note again that it is difficult to tell which one is true in a case when none of the conditions listed in Proposition 4.2 holds. At present, the best one can do is to try and construct a Legendrian representative with $t b+1=e$. If such a front is found, then we know that $\rho=M / 2$.)

Proposition 4.3. For a smooth knot type $K$, any of the following conditions implies that $\rho(K) \leq g(K)$.

(a) "Bennequin test": for the exponents defined above, we have $M \leq e$.

(b) "Conway test": the degree of the Conway polynomial $\nabla_{K}(z)=P_{K}(1, z)$ is $M$ or more.

Proof. There is nothing to prove if $\rho=-\infty$, so we will assume that $\rho=M / 2$. That implies $t b+1=e$ for those Legendrian representatives that have 2-graded rulings. Now, the Bennequin test is so called because it is obvious from (1-1) that

$$
\rho=\frac{M}{2} \leq \frac{e}{2}=\frac{t b+1}{2} \leq g .
$$


To prove the Conway test, recall that half the degree of the Conway polynomial is a lower bound for the genus, so in this case $\rho \leq M / 2 \leq g$.

Using the conditions of Propositions 4.2 and 4.3, Jim Hoste kindly conducted a computer search of the table of knots for potential examples with $\rho>g$. Knots with $M=0$ or 2 (that is, $\rho=0,1$ or $-\infty$ ) were also ruled out. Among prime knots up to 13 crossings, only two candidates were found: $13 n_{1426}$ and $13 n_{1456}$. It turns out that these knots do have 2-graded rulings; in fact $\rho=2$ for both, with unique genus 2 rulings. In both cases, it was possible to add a 1-handle to that ruling, realize the result as an embedded surface, and then find a Gabai disk decomposition [Gabai 1984]. Thus both knots have $g=3$. However at crossing number 14, the phenomenon of Example 1.3 occurs.

\section{Further research}

We expect to find deep links between the combinatorics of rulings and the geometry of Lagrangians in symplectic 4-space. If this prediction is correct, then some of the ideas outlined in this paper may even contribute to the solution of the GopakumarVafa conjecture. Let us use the following claim to illustrate further that it is useful to consider the topology of rulings.

Proposition 5.1. If a ruling is topologically a disk, then it is $\mathbb{Z}$-graded. In particular its boundary Legendrian has $r=0$.

Proof. The eyes of such a ruling are connected by the switches in a tree-like fashion. Consider a leaf of this tree and notice that because of the way the Maslov potential is defined, its single switch must be of index 0 . Then remove this eye from the diagram and induct on the number of eyes.

Remark 5.2. In fact, from the existence of a 2 -graded ruling it already follows that $r=0$ [Sabloff 2005]. On the other hand, there exist Legendrians with $r=0$ and ungraded rulings, hence with maximal $t b$ in their smooth type [Rutherford 2006], yet without a single 2-graded ruling. One such example is the untwisted double of the negative trefoil shown in Figure 4, produced using the method of [Kálmán 2008] on a +adequate diagram of the knot. From Proposition 3.5 it follows that no Legendrian representative of this knot can have a 2-graded ruling. (Similar examples can already be found among small alternating knots. The referee notes that $6_{2}$ has such a Legendrian representative.)

Finally, we wish to point out that other versions of ruling genus may equally well be introduced. To include multicomponent links, one can consider the minimum Euler characteristic $(\theta)$ of a ruling instead of maximum genus. For any nonnegative integer $\sigma$, one may define the class of $\sigma$-graded rulings [Fuchs 2003] and maximize 


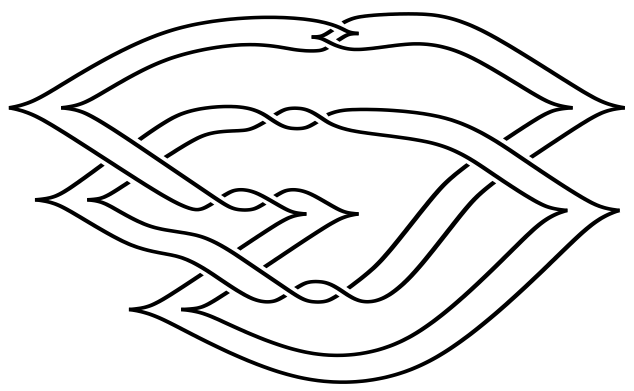

Figure 4. The untwisted double of the negative trefoil knot.

their Euler characteristic over all rulings and Legendrian representatives. Rutherford's work indicates that this link invariant may be most natural in the smooth category when $\sigma=1,2$ (see Remark 2.5). Other values of $\sigma$ may provide valuable information in the contact (Legendrian) category.

\section{Acknowledgements}

The phenomena described in this paper are related to Lagrangian surfaces in $B^{4}$ that a Legendrian in $S^{3}$ bounds. Thus this paper can be thought of as a prelude of the forthcoming joint work with Tobias Ekholm and Ko Honda [ $\geq 2008$ ]. I had many inspiring discussions with them. I also thank Jim Hoste for the tremendous insight gained from the computer searches of knot tables that he conducted for me. My conversations with Francis Bonahon and Dan Rutherford were very useful, too. Finally, I thank the referee for suggesting improvements to an early manuscript.

\section{References}

[Bennequin 1983] D. Bennequin, "Entrelacements et équations de Pfaff", pp. 87-161 in Third Schnepfenried geometry conference (Schnepfenried, 1982), vol. 1, Astérisque 107, Soc. Math. France, Paris, 1983. MR 86e:58070 Zbl 0573.58022

[Cromwell 2004] P. R. Cromwell, Knots and links, Cambridge University Press, Cambridge, 2004. MR 2005k:57011 Zbl 1066.57007

[Ekholm et al. $\geq 2008]$ T. Ekholm, K. Honda, and T. Kálmán, "Legendrian knots and Lagrangian cobordisms". In preparation.

[Etnyre 2005] J. B. Etnyre, "Legendrian and transversal knots", pp. 105-185 in Handbook of knot theory, edited by W. Menasco and M. Thistlethwaite, Elsevier B. V., Amsterdam, 2005. MR 2006j: $57050 \mathrm{Zbl} 05016798$

[Franks and Williams 1987] J. Franks and R. F. Williams, "Braids and the Jones polynomial", Trans. Amer. Math. Soc. 303:1 (1987), 97-108. MR 88k:57006 Zbl 0647.57002

[Fuchs 2003] D. Fuchs, "Chekanov-Eliashberg invariant of Legendrian knots: existence of augmentations", J. Geom. Phys. 47:1 (2003), 43-65. MR 2004h:57007 Zbl 1028.57005

[Gabai 1984] D. Gabai, "Foliations and genera of links", Topology 23:4 (1984), 381-394. MR 86h: 57006 Zbl 0567.57021 
[Kálmán 2008] T. Kálmán, "Maximal Thurston-Bennequin number of +adequate links”, Proc. Amer. Math. Soc. 136 (2008), 2969-2977.

[Morton 1986] H. R. Morton, "Seifert circles and knot polynomials", Math. Proc. Cambridge Philos. Soc. 99:1 (1986), 107-109. MR 87c:57006 Zbl 0588.57008

[Ng 2005] L. Ng, "A Legendrian Thurston-Bennequin bound from Khovanov homology", Algebr. Geom. Topol. 5 (2005), 1637-1653. MR 2007g:57027 Zbl 1091.57009

[Ng 2006] L. Ng, "On arc index and maximal Thurston-Bennequin number", preprint, 2006. arXiv math/0612356

[Pushkar' and Chekanov 2005] P. E. Pushkar' and Y. V. Chekanov, "Combinatorics of fronts of Legendrian links, and Arnol'd's 4-conjectures”, Uspekhi Mat. Nauk 60:1 (2005), 99-154. In Russian; translated in Russian Math. Surveys 60:1 (2005), 95-149 (with order of authors reversed). MR 2006h:58049 Zbl 1085.57008

[Rudolph 1990] L. Rudolph, "A congruence between link polynomials", Math. Proc. Cambridge Philos. Soc. 107:2 (1990), 319-327. MR 90k:57010 Zbl 0703.57005

[Rutherford 2006] D. Rutherford, "Thurston-Bennequin number, Kauffman polynomial, and ruling invariants of a Legendrian link: the Fuchs conjecture and beyond", Int. Math. Res. Not. 2006:15 (2006), Art. ID 78591. MR 2007a:57020 Zbl 1106.57012

[Sabloff 2005] J. M. Sabloff, "Augmentations and rulings of Legendrian knots", Int. Math. Res. Not. 2005:19 (2005), 1157-1180. MR 2006e:57010 Zbl 1082.57020

Received December 6, 2007. Revised March 14, 2008.

TAMÁS KÁLMÁN

UNIVERSITY OF TOKYO

Graduate SCHOOL of Mathematical SCIENCES

3-8-1 Komaba, Meguro-Ku

TOKYO, 153-8914

JAPAN

kalman@ms.u-tokyo.ac.jp

http://www.ms.u-tokyo.ac.jp 